\title{
Avaliação de correlação entre incidência, internação e mortalidade por COVID- 19 com o índice de Desenvolvimento Humano Municipal-IDHM em uma macrorregião de saúde de Minas Gerais
}

\author{
Assessment of correlation between incidence, hospitalization and mortality by COVID-19 with \\ the Municipal Human Development Index-IDHM in a health macro-region of Minas Gerais \\ Evaluación de la correlación entre incidencia, hospitalización y mortalidad por COVID-19 con \\ el Índice de Desarrollo Humano Municipal-IDHM en una macrorregión de salud de Minas
}

Gerais

Recebido: 04/01/2022 | Revisado: 09/01/2022 | Aceito: 11/01/2022| Publicado: 13/01/2022

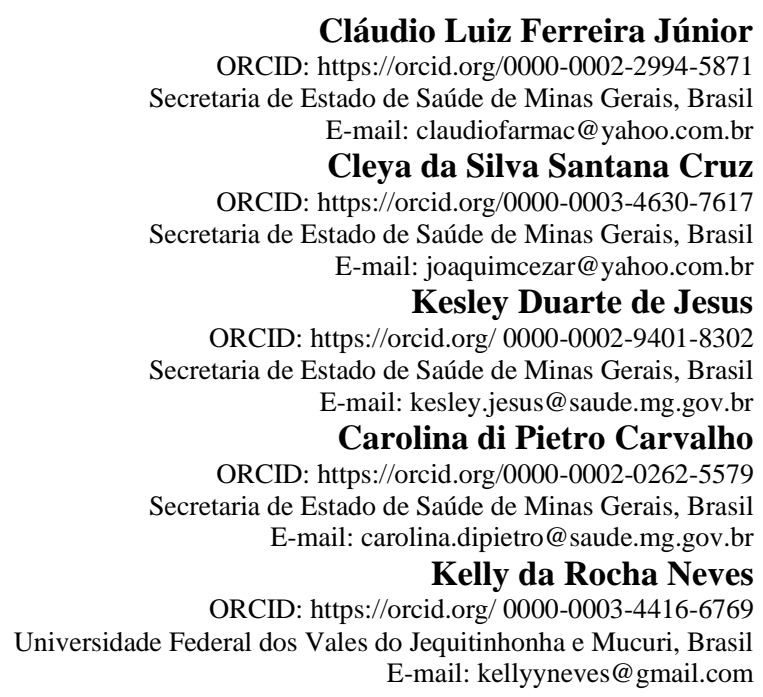

\section{Resumo}

A incidência da COVID-19 aconteceu de forma heterogênea em países, estados e municípios, dado as peculiaridades de cada nação, bem como as respostas de seus serviços de saúde em âmbito regional e local. O objetivo desse trabalho foi verificar a correlação entre o Índice de Desenvolvimento Humano Municipal (IDHM) e seus subíndices (renda, educação e longevidade) e as taxas de incidência, mortalidade e internação em uma macrorregião de saúde com 31 municípios. Os dados foram extraídos de bancos de dados online abertos, disponibilizados pelo Ministério da Saúde do Brasil e Secretaria de Estado de Saúde de Minas Gerais (SES-MG). Após aplicação de teste de normalidade sobre as taxas e IDHM, foi realizado teste de correlação de Spearman, a um nível de signficância de 5\%. Observou-se correlação positiva, moderada e significativa entre IDH/IDH renda com taxa de incidência e taxa de mortalidade, evidenciando que o aumento de IDH foi associado a incidência de COVID-19 e também mortalidade. A hipótese principal formulada foi que municípios com maiores IDH podem favorecer uma maior circulação de pessoas, bens e serviços, que impactam diretamente na dinâmica de disseminação do vírus. Espera-se que mais trabalhos possam ser realizados visando buscar correlação entre indicadores socioeconômicos, incidência e mortalidade da COVID-19, fortalecendo e direcionando os processos de vigilância nos vários níveis de saúde.

Palavras-chave: COVID-19; Índice de desenvolvimento humano; Renda; Mortalidade; Incidência.

\begin{abstract}
The incidence of COVID-19 was heterogeneous in countries, states and municipalities, given the peculiarities of each nation, as well as the responses of their health services at regional and local levels. The objective of this work was to verify the correlation between the Municipal Human Development Index (MHDI) and its sub-indices (income, education and longevity) and the incidence, mortality and hospitalization rates in a health macro-region with 31 municipalities. Data were extracted from open online databases, made available by the Ministry of Health of Brazil and
\end{abstract}


the State Health Department of Minas Gerais (SES-MG). After applying the normality test on rates and MHDI, Spearman's correlation test was performed, at a significance level of 5\%. A positive, moderate and significant correlation was observed between HDI/HDI income with incidence rate and mortality rate, showing that the increase in HDI was associated with the incidence of COVID-19and also mortality. The main hypothesis formulated was that municipalities with higher HDI can favor a greater circulation of people, goods and services, which directly impact the dynamics of virus dissemination. It is expected that more work can be carried out to seek correlation between socioeconomic indicators, incidence and mortality of COVID-19, strengthening and directing surveillance processes at various levels of health.

Keywords: COVID-19; Human development index; Income; Mortality; Incidence.

\section{Resumen}

La incidencia de COVID-19 fue heterogénea en países, estados y municipios, dadas las peculiaridades de cada nación, así como las respuestas de sus servicios de salud a nivel regional y local. El objetivo de este trabajo fue verificar la correlación entre el Índice de Desarrollo Humano Municipal (IDHM) y sus subíndices (ingreso, educación y longevidad) y las tasas de incidencia, mortalidad y hospitalización en una macrorregión de salud con 31 municipios. Los datos fueron extraídos de bases de datos abiertas en línea, disponibles por el Ministerio de Salud de Brasil y el Departamento de Salud del Estado de Minas Gerais (SES-MG). Luego de aplicar la prueba de normalidad sobre tasas y IDHM, se realizó la prueba de correlación de Spearman, a un nivel de significancia del 5\%. Se observó una correlación positiva, moderada y significativa entre los ingresos del IDH / IDH con la tasa de incidencia y la tasa de mortalidad, mostrando que el aumento del IDH se asoció con la incidencia de COVID-19 y también con la mortalidad. La principal hipótesis formulada fue que los municipios con mayor IDH pueden favorecer una mayor circulación de personas, bienes y servicios, lo que impacta directamente en la dinámica de diseminación del virus. Se espera que se pueda trabajar más para buscar la correlación entre indicadores socioeconómicos, incidencia y mortalidad de COVID-19, fortaleciendo y dirigiendo los procesos de vigilancia en los distintos niveles de salud.

Palabras clave: COVID-19; Índice de desarrollo humano; Ingreso; Mortalidad; Incidencia.

\section{Introdução}

Em 31 de dezembro de 2019, a Organização Mundial de Saúde (OMS) foi alertada sobre vários casos de pneumonia na cidade de Wuhan, província de Hubei, na República Popular da China. No Brasil, em 3 de fevereiro de 2020, o Ministério da Saúde (MS) declarou, a infecção pelo novo coronavírus (SARS-CoV-2) pelo Decreto Federal n ${ }^{\text {o }}$ 7.616/2011, emergência de Saúde Pública Nacional. Em 11 de março de 2020, a OMS, classificou a pandemia pelo Coronavírus - COVID-19 uma situação emergente e em rápida evolução, uma doença altamente infecciosa, com casos assintomáticos e com sintomas clínicos principais que incluem febre, tosse, fadiga, mialgias, dispneia. Uma Síndrome Gripal, que quando grave evolui para Síndrome do Desconforto Respiratório Agudo Grave - SRAG, resultando em óbito (Malta et al., 2020; Brasil, 2021).

Medidas sanitárias como etiqueta respiratória, distanciamento social, higienização das mãos, uso de álcool, de máscara e a vacinação têm sido empregados no mundo e no Brasil para mitigar a Pandemia de COVID-19, uma vez que até o momento ainda não há evidências científicas que garantam eficácia terapêutica de medicamentos específicos (Dourado et al., 2020; Soares et al., 2021)

Nota-se que essas medidas, nesse processo de ascensão da COVID-19, as nações apresentaram índices de contágio em maior ou menor grau, proporções heterogêneas que representaram um novo perfil epidemiológico da doença. Como premissa nesta abordagem, está o índice de desenvolvimento humano - IDH que é aferido a partir de uma média geométrica de índices que medem cada um dos fatores como educação, longevidade e renda. Assim, como em alguns países com economia heterogênea, brasileiros vivem em regiões com ausência de saneamento básico e densamente povoadas, apresentando diferença no processo epidêmico da COVID-19 em macrorregiões de saúde (Maciel et al, 2020).

Pandemias raramente afetam todas as pessoas de uma forma uniforme, assim bem como o risco de infecção e óbito nas diferentes camadas sociais e localidades (Dourado et al., 2020; Henriques \& Vasconcelos, 2020). A Macrorregião de Saúde Jequitinhonha possui em sua jurisdição 31 municípios com características cultural, social, demográficas, econômicas diversificadas como atividade extrativista (mineração, agropecuária e outras) e o turismo ecológico, circuito da estrada real, com predomínio de características rurais, transição de bioma entre cerrado e mata atlântica dentro dessa dimensão geográfica, entre 
outros fatores, e que confere aos serviços desafios para mitigar a Pandemia. Neste contexto, o presente estudo busca identificar a correlação entre índice de desenvolvimento humano - IDH com a incidência, mortalidade e internação clínica por COVID-19 nesta macrorregião de saúde.

\section{Metodologia}

Foi realizado estudo do tipo observacional, analítico, ecológico de abordagem quantitativa (Lima-Costa \& Barreto, 2003), de casos, óbitos e internações clínicas por COVID-19 nos municípios da Macrorregião de Saúde Jequitinhonha entre março de 2020 e novembro de 2021.

A Macrorregião de Saúde Jequitinhonha é uma das 14 macrorregiões de saúde do estado de Minas Gerais. É composta por 31 municípios, distribuídos em um território de 27.718,0 Km², com uma população estimada de 408.352 habitantes (Minas Gerais, 2019).

Os dados estudados referentes à COVID-19 foram coletados na Plataforma de Informações em Saúde (TABNET) do Departamento de Informática do Sistema Único de Saúde (DATASUS) (Brasil, 2021) e do Painel de Monitoramento COVID19 da Secretaria de Estado da Saúde de Minas Gerais (SES/MG) (Minas Gerais 2021), sendo dados secundários e abertos, de domínio público. As informações sociais dos municípios foram coletadas no Atlas de Desenvolvimento Humano no Brasil (Brasil, 2013), sistematizadas pelo Programa das Nações Unidas para o Desenvolvimento (PNUD) de 2010. A estimativa oficial da população de cada município da macrorregião Jequitinhonha, foi obtida para o ano de 2021, Instituto Brasileiro de Geografia e Estatística (IBGE) e Tribunal de Contas da União (Minas Gerais, 2021a).

Por se tratar de uma pesquisa cujos bancos de dados são de domínio público, disponíveis na rede de internet, cedidos pelo Ministério da Saúde e Secretaria de Estado da Saúde de Minas Gerais (SES/MG) para livre consulta, e garantido o sigilo dos seres humanos envolvidos, não foi necessária a submissão, ao Comitê de Ética em Pesquisa (Brasil, 2012).

Os casos confirmados de COVID-19 foram tabulados no painel de monitoramento de casos, na aba perfil geográfico, casos, selecionando macrorregião/municípios. Da mesma forma, o quantitaivo de óbitos por COVID-19 também foi tabulado considerando o painel diário do monitoramento de casos da SES/MG, na aba perfil geográfico, óbitos, selecionando macrorregião/municípios. Os dados foram coletados em 30 e novembro de 2021. É importante destacar que o painel de monitoramento COVID-19 do estado de Minas Gerais é alimentado diariamente.

Para a coleta dos casos de internações clínicas por COVID-19 na base do DATASUS foi utilizado o filtro aba assistência à Saúde Produção Hospitalar, Dados Detalhados de AIH (SP), por local de internação por estado, macrorregião de saúde/município, tendo como procedimento tratamento de infecção pelo coronavírus - COVID-19(0303010223), procedimentos clínicos, de média complexidade.

$\mathrm{Na}$ análise de dados foi realizada estatística descritiva com os dados dos casos e da população de cada município, sendo em seguida feito o cálculo incidência, internação e mortalidade por COVID-19, todas por município de residéncia. A variável IDH foi apresentada pelos respectivos valores por município, referente aos três subíndices de IDH: renda, educação e longevidade.

Foram consideradas variáveis dependentes, a taxa de incidência da COVID-19 por município, taxa de mortalidade por COVID-19 por município e taxa de internação clínica por município. Para cálculo da taxa de incidência acumulada, dividiu-se o total de casos do período pela população geral, multiplicada por 100 mil habitantes. A taxa de mortalidade por COVID-19 foi calculada dividindo-se o número de óbitos no período pela população, multiplicado por 100 mil habitantes. E para determinar a taxa de internação em leitos clínicos, o número de internações no ano foi dividido pela população no mesmo período, multiplicado por 10 mil.

Como variável independente foi utilizada o IDHM, sendo a variável IDH estimada para cada município no ano de 2010, 
conforme disponível no PNUD. E os seus três subíndices, educação, renda e longevidade para comparar o nível geral alcançado e a incidência, óbitos e internações por COVID-19.

Para o teste de normalidade da amostra foi utilizado Shapiro-Wilk, e como a amostra não apresentou distribuição normal, foi utilizada a correlação de Spearman. Sendo consideradas estatisticamente significativas quando o valor de $\mathrm{p}<0,05$.

A correlação de Spearman foi utilizada para investigar associações entre a taxa de incidência da doença, mortalidade e internação em leitos clínicos com os IDH municipais, educação, longevidade e renda para os municípios (n=31) que compõem Macrorregião de Saúde do Jequitinhonha.

Os valores de correlação gerados pelas taxas e IDH puderam ser interpretados, segundo classificação se Spearman, como positivas ou negativas e como muito fraca $(0,00$ a 0,19$)$ fraca $(0,20$ a 0,39$)$, moderada $(0,40$ a 0,69$)$, forte $(0,70$ a 0,89$)$, ou muito forte $(0,90$ a 1,00$)$.

Os dados coletados foram preenchidos no editor de planilhas do programa Excel®, com correspondência de todas as informações para formulação do banco de dados completo no programa Sistema R (R version 4.0.5 (2021-03-31), onde foram realizadas as análises estatísticas.

\section{Resultados e Discussão}

A Tabela 1 mostra a taxa de incidência, de mortalidade e internação para a população de cada município da Macrorregião Jequitinhonha, a taxa de incidência da doença na Macrorregião foi de 6.478/100.000 habitantes. As maiores incidências ocorreram nos municípios de Conceição do Mato Dentro (18.293,4/100.000 hab.), Datas (11.175,0/100.000 hab.) e Diamantina (10.671,1/100.000 hab.), que foram maiores que a incidência do estado de Minas Gerais (10.404,20/100.000 hab.).

A taxa de internação por COVID-19 em leitos clínicos na Macrorregião foi de 52,52/10.000 hab. Os municípios que apresentaram maiores taxas de internações foram: Gouveia (156,6/10.000 hab.), Datas (142,7/10.000 hab.) e Diamantina (115/10.000 hab.). A Macrorregião apresentou taxa de mortalidade por COVID-19 de 111,52/100.000 hab., enquanto que a taxa de mortalidade em Minas Gerais foi de 264,7/100.000 hab. Os municípios da macrorregião com maior mortalidade por COVID19 foram: Gouveia (287,9/100.000 hab.), Datas (257,6/100.000 hab.), Conceição do Mato Dentro (217, 9/100.000 hab.) e Diamantina (204,5/100.000 hab.).

Tabela 1 - Relação das taxas de incidência por 100.000 habitantes, mortalidade por 100.00 habitantes e internação por 10.000 habitantes, por COVID-19 dos municípios (n=31) da Macrorregião de Saúde do Jequitinhonha.

(Continua)

\begin{tabular}{|c|c|c|c|c|}
\hline Município & Taxa de Incidência & Taxa de mortalidade & Taxa de Internação & População \\
\hline Alvorada de Minas & 8155,3 & 83,2 & 91,5 & 3605 \\
\hline Araçuaí & 5986,7 & 147,1 & 33,8 & 36715 \\
\hline Aricanduva & 3053,7 & 0,0 & 11,3 & 5.305 \\
\hline Berilo & 1295,2 & 8,5 & 5,1 & 11813 \\
\hline Capelinha & 8123,5 & 93,9 & 41,5 & 38321 \\
\hline Carbonita & 7322,5 & 63,7 & 29,7 & 9423 \\
\hline Chapada do Norte & 1532,5 & 19,6 & 13,7 & 15334 \\
\hline Coluna & 6015,9 & 113,5 & 55,6 & 8810 \\
\hline Conceição do Mato Dentro & 18293,4 & 217,9 & 49,3 & 17438 \\
\hline Congonhas do Norte & 1902,1 & 79,3 & 49,5 & 5047 \\
\hline Coronel Murta & 4148,1 & 76,0 & 26,1 & 9209 \\
\hline
\end{tabular}


Research, Society and Development, v. 11, n. 1, e50111125351, 2022

(CC BY 4.0) | ISSN 2525-3409 | DOI: http://dx.doi.org/10.33448/rsd-v11i1.25351

\begin{tabular}{|c|c|c|c|c|}
\hline & & & \multicolumn{2}{|c|}{ (Conclusão) } \\
\hline Couto de Magalhães de Minas & 3651,9 & 157,8 & 58,6 & 4436 \\
\hline Datas & 11175,0 & 257,6 & 142,7 & 5047 \\
\hline Diamantina & 10671,1 & 204,5 & 115,0 & 47924 \\
\hline Felício dos Santos & 5111,7 & 107,4 & 64,4 & 4656 \\
\hline Francisco Badaró & 1609,9 & 58,2 & 7,8 & 10311 \\
\hline Gouveia & 6121,4 & 287,9 & 156,6 & 11811 \\
\hline Itamarandiba & 6598,3 & 79,7 & 39,3 & 35130 \\
\hline Jenipapo de Minas & 2364,7 & 64,3 & 20,6 & 7781 \\
\hline José Gonçalves de Minas & 3062,1 & 22,4 & 22,4 & 4474 \\
\hline Leme do Prado & 3636,0 & 60,9 & 20,3 & 4923 \\
\hline Minas Novas & 2840,5 & 79,3 & 54,9 & 31509 \\
\hline Presidente Kubitschek & 7533,3 & 233,3 & 63,3 & 3000 \\
\hline Santo Antônio do Itambé & 2949,8 & 26,6 & 34,5 & 3763 \\
\hline São Gonçalo do Rio Preto & 7457,5 & 157,3 & 50,3 & 3178 \\
\hline Senador Modestino Gonçalves & 4881,7 & 197,2 & 71,5 & 4056 \\
\hline Serra Azul de Minas & 4007,5 & 0,0 & 32,6 & 4292 \\
\hline Serro & 6010,0 & 86,1 & 62,6 & 20915 \\
\hline Turmalina & 7904,3 & 108,5 & 50,8 & 20280 \\
\hline Veredinha & 4988,7 & 17,4 & 29,7 & 5733 \\
\hline Virgem da Lapa & 4486,9 & 43,7 & 15,3 & 13729 \\
\hline
\end{tabular}

Fonte: Painel de monitoramento Covid-19 (2021) e TCU (2021).

A Tabela 2 evidencia o IDH municipal e seus indicadores ligados à renda, educação e longevidade. Observa-se que como maior IDH municipal, têm-se os municípios de Diamantina $(0,716)$, Turmalina $(0,682)$ e Gouveia $(0,681)$, e os menores IDH foram Serra Azul de Minas (0,557), Santo Antônio do Itambé $(0,558)$ e Congonhas do Norte $(0,568)$.

Tabela 2 - Índice de Desenvolvimento Humano Municipal (IDHM) e seus três principais indicadores: Educação, Saúde e Renda dos municípios (n=31) da Macrorregião de Saúde Jequitinhonha.

(Continua)

\begin{tabular}{|c|c|c|c|c|}
\hline Município & IDHM & IDHM renda & IDHM Longevidade & IDHM Educação \\
\hline Alvorada de Minas & 0,572 & 0,57 & 0,794 & 0,414 \\
\hline Araçuaí & 0,663 & 0,633 & 0,821 & 0,560 \\
\hline Aricanduva & 0,582 & 0,553 & 0,787 & 0,454 \\
\hline Berilo & 0,628 & 0,580 & 0,816 & 0,524 \\
\hline Capelinha & 0,653 & 0,641 & 0,824 & 0,527 \\
\hline Carbonita & 0,638 & 0,617 & 0,796 & 0,529 \\
\hline Chapada do Norte & 0,598 & 0,571 & 0,798 & 0,469 \\
\hline Coluna & 0,583 & 0,590 & 0,782 & 0,430 \\
\hline Conceição do Mato Dentro & 0,634 & 0,622 & 0,794 & 0,517 \\
\hline
\end{tabular}




\begin{tabular}{|c|c|c|c|c|}
\hline Congonhas do Norte & 0,568 & 0,566 & 0,746 & 0,433 \\
\hline Coronel Murta & 0,627 & 0,578 & 0,784 & 0,543 \\
\hline Couto de Magalhães de Minas & 0,659 & 0,600 & 0,807 & 0,592 \\
\hline Datas & 0,616 & 0,590 & 0,756 & 0,524 \\
\hline Diamantina & 0,716 & 0,693 & 0,839 & 0,632 \\
\hline Felício dos Santos & 0,606 & 0,568 & 0,794 & 0,494 \\
\hline Francisco Badaró & 0,622 & 0,575 & 0,793 & 0,527 \\
\hline Gouveia & 0,681 & 0,622 & 0,833 & 0,609 \\
\hline Itamarandiba & 0,646 & 0,618 & 0,798 & 0,547 \\
\hline Jenipapo de Minas & 0,624 & 0,588 & 0,793 & 0,520 \\
\hline José Gonçalves de Minas & 0,632 & 0,597 & 0,764 & 0,553 \\
\hline Leme do Prado & 0,670 & 0,602 & 0,779 & 0,640 \\
\hline Minas Novas & 0,633 & 0,600 & 0,802 & 0,528 \\
\hline Presidente Kubitschek & 0,595 & 0,573 & 0,787 & 0,468 \\
\hline Santo Antônio do Itambé & 0,558 & 0,537 & 0,794 & 0,408 \\
\hline São Gonçalo do Rio Preto & 0,640 & 0,593 & 0,815 & 0,542 \\
\hline Senador Modestino Gonçalves & 0,620 & 0,566 & 0,782 & 0,539 \\
\hline Serra Azul de Minas & 0,557 & 0,537 & 0,809 & 0,398 \\
\hline Serro & 0,656 & 0,650 & 0,834 & 0,520 \\
\hline Turmalina & 0,682 & 0,646 & 0,816 & 0,602 \\
\hline Veredinha & 0,632 & 0,579 & 0,785 & 0,555 \\
\hline Virgem da Lapa & 0,610 & 0,585 & 0,797 & 0,488 \\
\hline
\end{tabular}

Fonte: Autores.

Quanto à correlação, observou-se associação positiva entre a taxa de incidência (rho = 0,338, p< 0,05) e mortalidade (rho $=0,392, \mathrm{p}<0,05)$ com o IDHM, e, especificamente, essa correlação é influenciada pelo IDH renda para ambas as taxas: taxa de incidência (rho $=0,498, \mathrm{p}<0,05)$ e taxa de mortalidade (rho= 0, 459, p <0,05), correlações moderadas. A tabela 3 e os Gráficos de dispersão 1 e 2 evidenciam essas informações.

Tabela 3- Correlação entre taxa de incidência, mortalidade e internação com Índice de Desenvolvimento Humano Municipal (IDH) municipal, educação, longevidade e renda. Correlação de Spearman, Rho ( $\rho)$.

\begin{tabular}{lllll}
\hline Taxas & IDHM & IDH Educação & IDH Longevidade & IDH renda \\
\hline Taxa de Incidência & $0,338^{*}$ & 0,175 & 0,224 & $0,498^{*}$ \\
Taxa de Mortalidade & $0,392^{*}$ & 0,289 & 0,167 & $0,459^{*}$ \\
Taxa de Internação & 0,154 & 0,081 & 0,155 & 0,230 \\
\hline
\end{tabular}

$* \mathrm{p}<0,05$. Fonte: Autores (2021) e Atlas de Desenvolvimento Humano no Brasil (2013). 
Gráfico 1 - Diagrama de dispersão de Índice de Desenvolvimento Humano Municipal (IDH) (Renda) pela Incidência de COVID-19(por 100.000 habitantes). Rho $=0,498, \mathrm{p}<0,05$.

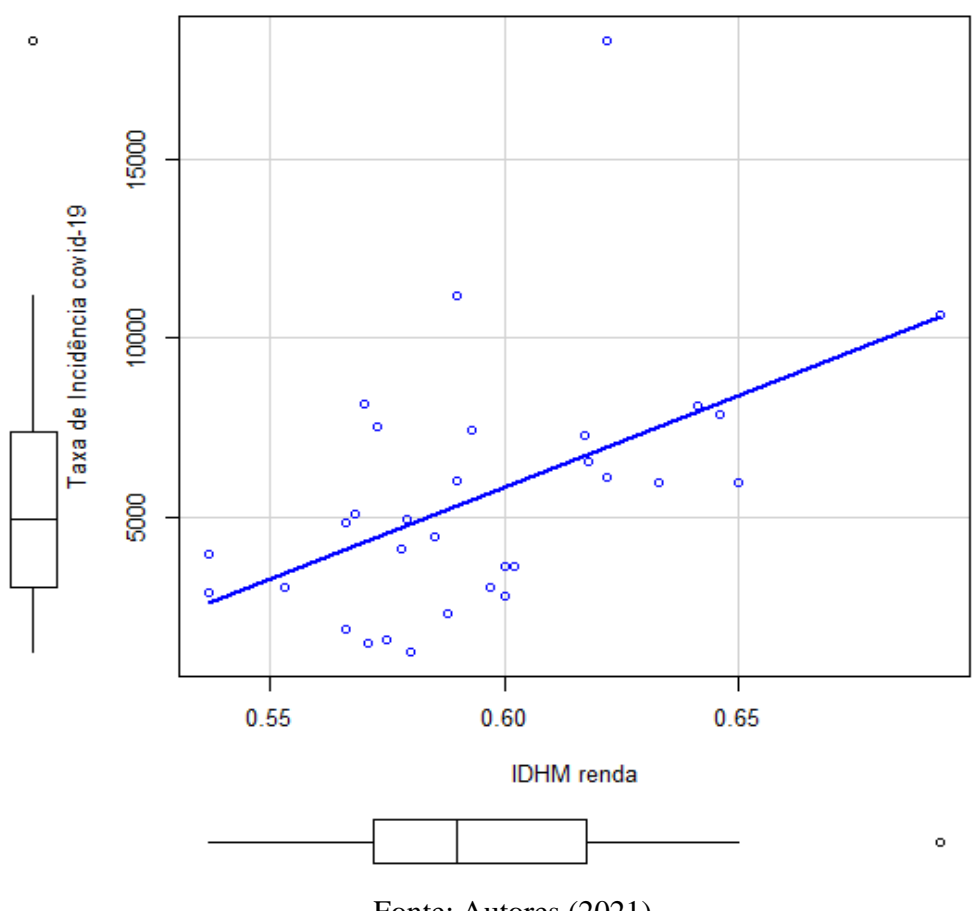

Fonte: Autores (2021).

Gráfico 2 - Diagrama de dispersão de Índice de Desenvolvimento Humano Municipal (IDH) (Renda) pela Mortalidade por COVID-19(por 100.000 habitantes). Rho $=0,459, \mathrm{p}<0,05$.

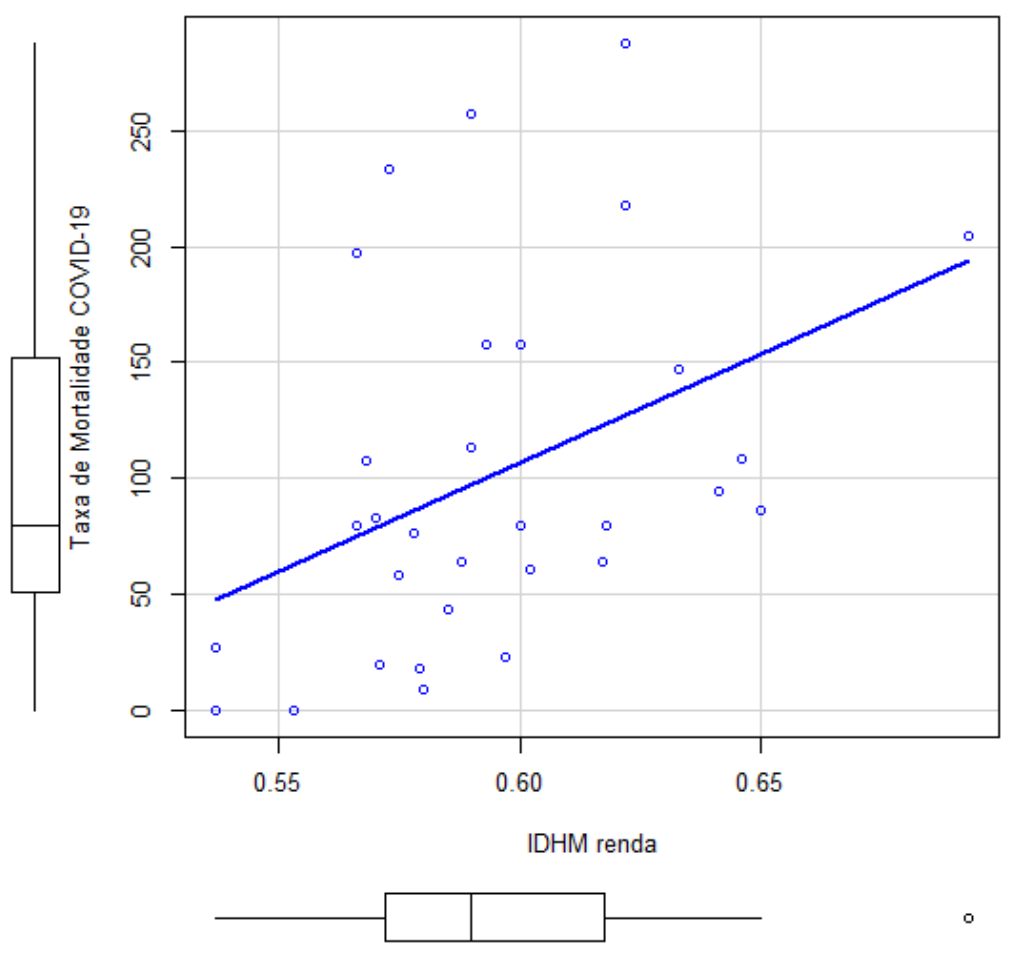

Fonte: Autores (2021). 
O Brasil apresenta proporções econômicas heterogêneas e desigualdade no acesso aos serviços de saúde, o que contribui para maior quantitativo de casos da COVID-19 e enfatiza a importância de monitorar o perfil epidemiológico da doença e a oferta dos serviços públicos de saúde (Nunes \& Pan-American Health Organization, 2001; Barbalho et al. 2021). Poucos trabalhos até o momento se debruçaram em investigar a relação do IDH com a COVID-19, e não havendo uma concordância entre eles. Neste estudo pode-se observar uma correlação positiva entre taxas de incidência e mortalidade a maiores IDHM e seu subitem, renda, nessa região de saúde.

De maneira semelhante, o aumento do IDH foi associado a um aumento da incidência da doença nas Regiões das Américas e Africana e a um aumento da mortalidade nas Regiões das Américas, Europeia e Africana (Torkian et al., 2020). Dourado et al. (2021) também encontraram correlação positiva entre a incidência da doença e IDH maiores. As hipóteses formuladas por esses autores para explicar esses achados, corroboradas por este estudo, são de que as cidades menores, que frequentemente têm menores IDH, apresentam menos estruturas e estabelecimentos que favoreçam aglomerações e, por conseguinte, o contágio da doença.

Estudo na Itália também mostrou uma correlação positiva entre IDH e incidência e letalidade da COVID-19, os autores classificaram como inesperado esse resultado, no entanto atribuíram esses achados a um maior número de pessoas vivendo com doenças crônicas nesses locais (Liu et al., 2020).

Nesse ponto, partimos também da hipótese de que municípios com IDH renda maiores realizaram mais testes, aumentando a detecção da doença e por consequência a taxa de incidência. Como exemplo, países com maiores IDH têm maior capacidade de investirem em seus sistemas de saúde, podem implantar sistemas de vigilância mais desenvolvidos e notificar casos e óbitos por COVID-19 com maior eficiência (Squalli, 2020). Esse raciocínio pode ser transposto para as realidades municipais. Áreas mais desenvolvidas possuem sistema de saúde relativamente eficaz (Hu, 2013).

Correlação positiva, moderada e significativa também foi encontrada em estudo que investigou a relação da incidência da COVID-19 com o IDH nos municípios do Ceará (Maciel et al., 2020). No Rio Grande do Sul, A incidência da COVID-19, em média, foi maior naqueles municípios com índice IDH mais alto, e esses valores, segundo os autores, podem ser explicados pelo fato de que as maiores regiões do Rio Grande do Sul, como Porto Alegre e Caxias do Sul, que tem maiores valores de incidência, também tem maior IDH (Conte, 2020).

Os achados citados acima demonstram que os resultados deste estudo não são exceção. No intuito de compreender melhor estes achados, foram testados os subitens do IDHM o que apontou correlação positiva e moderada com o item renda, sugerindo que este seja o maior responsável pela correlação positiva entre as variáveis dependentes e independentes. Nesse sentido, a hipótese levantada por outros pesquisadores, em outros estudos, de que municípios mais desenvolvidos economicamente e que, portanto, oferecem mais serviços, possuem maior circulação de bens e pessoas e por isso propiciam uma maior circulação do vírus, também pode servir como modelo explicativo para este trabalho (Conte, 2020; Squalli, 2020; Dourado et al 2021).

Um exemplo a essa hipótese é o município com maior IDH, apresentado neste estudo. O município de Diamantina apresentou as maiores taxas de incidência e mortalidade dentre os 31 municípios da Macrorregião Jequitinhonha. Este é também o município de maiores índices de renda e longevidade e não por acaso é o polo de serviços de saúde para a região, abrigando inclusive a sede da Superintendência Regional de Saúde. Em seu território localiza-se ainda a Universidade Federal dos Vales do Jequitinhonha e Mucuri, uma das primeiras instituições credenciadas para realizar testes de detecção da COVID-19 no Estado, logo no início da pandemia. Além disso, trata-se de uma cidade turística, reconhecida internacionalmente, Patrimônio Cultural de Humanidade, e que por isso atrai muitos visitantes. Dessa forma, dentro da macrorregião pesquisada, este pode ser considerado um município com alta circulação de pessoas e produtos, além de ofertar diversos serviços essenciais para os demais municípios 
da região que não puderam ser paralisados durante os momentos mais críticos da pandemia, o que pode ter contribuído para disseminação e desfecho da doença.

Apesar da correlação positiva entre renda e taxas de incidência e mortalidade por COVID-19 nesses municípios, é necessário ressaltar que estes dados não permitem concluir que indivíduos de maior renda foram mais afetados pela doença. No Brasil, a concentração de renda é extremamente elevada, o que significa que, em territórios considerados "ricos" pode se abrigar uma população extremamente pobre. Nos estados brasileiros, 59,8\% da variação da incidência de COVID-19 foi justificada pela desigualdade de renda (Figueiredo et al., 2020), indicando a importância que esse fator teve no isolamento e circulação do vírus.

\section{Conclusão}

O presente trabalho, que se baseou em fontes de dados oficiais mostrou correlação positiva e significativa entre IDH/ IDH renda e incidência por COVID-19, e entre IDH/IDH renda e mortalidade por COVID-19. Algumas hipóteses podem explicar esses achados, como a alta incidência e consequente mortalidade ligadas a locais onde houve maior probabilidade da circulação do vírus, que seriam cidades onde ofertam um maior número de serviços e, portanto, estão mais propícias a criar um cenário de contaminação. A importância de avaliar também que municípios com maiores IDH podem estar associados a maiores ações de testagem e, portanto, com menos casos subnotificados; seus sistemas de vigilância podem ter agido mais rápido. Para além das hipóteses formuladas, a busca de relação entre indicadores socioeconômicos e taxas relacionadas à COVID-19 pode contribuir para identificar lacunas e fortalecer ações de enfrentamento regional à pandemia.

Como sugestão para trabalhos futuros fica a necessidade de se investigar essas associações em outras regiões do estado de Minas Gerais e ampliar o número de indicadores para busca de correlação como, por exemplo, a utilização do Índice Gini, que é um instrumento para medir o grau de concentração de renda, complementar ao IDH renda.

\section{Referências}

Barbalho, E. de V., Gomes, M. L. F., Pereira, T. C. C., Pedrosa, E. C. R., Linard, C. F. B. M., \& Pinto, F. J. M. (2021). Indicadores sociodemográficos na pandemia da COVID-19por meio da distribuição espacial no Brasil: Revisão integrativa. Research, Society and Development, $10(6)$, e34110615507. https://doi.org/10.33448/rsd-v10i6.15507

Brasil, Ministério da Saúde. (2012). Resolução N o 466, dE 12 de dezembro de 2012 (Vol. 66, Issue December).

Brasil, Secretaria de Vigilância em Saúde (MS). Guia de vigilânciaepidemiológica Emergência de saúde pública de Importância nacional pela Doença pelo coronavírus 2019 - COVID-19. Departamento de Análise em Saúde e Doenças não Transmissíveis. Brasília, 2021. 86p.

Brasil. Atlas de Desenvolvimento Humano no Brasil [Internet]. 2013 [acessado em 7 maio 2020]. Disponível em: http://atlasbrasil.org.br/2013/pt/consulta/

Brasil. Ministério da Saúde. (2021). Departamento de Informática do Sistema Único de Saúde http://tabnet.datasus.gov.br/cgi/deftohtm.exe?sih/cnv/spamg.def

Conte, B. P. (2020). A INCIDÊNCIA DA COVID-19NOS MUNICÍPIOS GAÚCHOS: TERIAM TODOS CONDIÇÕES DE COMBATER O AVANÇO DO VÍRUS? Observatório Socioeconômico da COVID-19.

Dourado, P., Rodrigues, P., Vieira, L., \& Lima, A. ([s.d.]). RELAÇÃO DA COVID-19COM O ÍNDICE DE DESENVOLVIMENTO HUMANO - IDH. 12. Brasília, 2020.

Figueiredo, A. M. de, Figueiredo, D. C. M. M. de, Gomes, L. B., Massuda, A., Gil-García, E., Vianna, R. P. de T., \& Daponte, A. (2020). Social determinants of health and COVID-19infection in Brazil: An analysis of the pandemic. Revista Brasileira de Enfermagem, 73 (suppl 2), e20200673. https://doi.org/10.1590/0034-7167-2020-0673

Henriques, C. M. P., \& Vasconcelos, W. (2020). Crises dentro da crise: Respostas, incertezas e desencontros no combate à pandemia da COVID-19 no Brasil. Estudos Avançados, 34(99), 25-44. https://doi.org/10.1590/s0103-4014.2020.3499.003

Hu, Q.-D. (2013). Human development index is associated with mortality-to-incidence ratios of gastrointestinal cancers. World Journal of Gastroenterology, 19(32), 5261. https://doi.org/10.3748/wjg.v19.i32.5261

Lima-Costa, M. F., \& Barreto, S. M. (2003). Tipos de estudos epidemiológicos: Conceitos básicos e aplicações na área do envelhecimento. Epidemiologia e Serviços de Saúde, 12(4). https://doi.org/10.5123/S1679-49742003000400003

Liu, K., He, M., Zhuang, Z., He, D., \& Li, H. (2020). Unexpected positive correlation between human development index and risk of infections and deaths of COVID-19in Italy. One Health, 10, 100174. https://doi.org/10.1016/j.onehlt.2020.100174

Maciel, J. A. C., Castro-Silva, I. I., \& Farias, M. R. de. (2020). Análise inicial da correlação espacial entre a incidência de COVID-19 e o desenvolvimento 
Research, Society and Development, v. 11, n. 1, e50111125351, 2022

(CC BY 4.0) | ISSN 2525-3409 | DOI: http://dx.doi.org/10.33448/rsd-v11i1.25351

humano nos municípios do estado do Ceará no Brasil. Revista Brasileira de Epidemiologia, 23, e200057. https://doi.org/10.1590/1980-549720200057

Malta, D. C., Szwarcwald, C. L., Barros, M. B. de A., Gomes, C. S., Machado, Í. E., Souza Júnior, P. R. B. de, Romero, D. E., Lima, M. G., Damacena, G. N., Pina, M. de F., Freitas, M. I. de F., Werneck, A. O., Silva, D. R. P. da, Azevedo, L. O., \& Gracie, R. (2020). A pandemia da COVID-19 e as mudanças no estilo de vida dos brasileiros adultos: Um estudo transversal, 2020. Epidemiologia e Serviços de Saúde, 29(4), e2020407. https://doi.org/10.1590/s167949742020000400026

Minas Gerais 2021. Painel de Monitoramento COVID-19. https://coronavirus.saude.mg.gov.br/painel

Minas Gerais, 2021a. Regionalização. https://saude.mg.gov.br/parceiro/regionalizacao-pdr2

Minas Gerais. Secretaria de Estado da Saúde de Minas Gerais. Deliberação CIBSUS/MG No 3.013, de 23 de outubro de 2019. Aprova o Ajuste/2019 do Plano Diretor de Regionalização PDR/SUSMG e dá outras providências. Belo Horizonte, 2019. Imprensa Oficial do Estado de Minas Gerais, Belo Horizonte, MG, 24 out. 2019.

Nunes, A., \& Pan-American Health Organization (Orgs.). (2001). Medindo as desigualdades em saúde no Brasil: Uma proposta de monitoramento (1a. ed). OPAS/OMS.

Soares, K. H. D., Oliveira, L. da S., Da Silva, R. K. F., Silva, D. C. de A., Farias, A. C. do N., Monteiro, E. M. L. M., \& Compagnon, M. C. (2021). Medidas de prevenção e controle da COVID-19: Revisão integrativa. Revista Eletrônica Acervo Saúde, 13(2), e6071. https://doi.org/10.25248/reas.e6071.2021

Squalli, J. (2020). Evaluating the determinants of COVID-19 mortality: A cross-country study. medRxiv, 2020.05.12.20099093. https://doi.org/10.1101/2020.05.12.20099093

Torkian, S., Kazemi, S., Eslahi, M., Khatooni, E., Etesami, R., Khanjani, N., \& Rashti, R. (2020). The Association Between COVID-19Incidence and Mortality with Socioeconomic Development—A Global Ecological Study [Preprint]. In Review. https://doi.org/10.21203/rs.3.rs-117325/v1 